\title{
Breast cancer epidemiology among surgically treated patients in Jordan: A retrospective study
}

\author{
Hala Halbony ${ }^{1}$ (D) Khadija Salman², Ahmad Alqassieh², Mutaz Albrezat ${ }^{2}$,Ahmad Hamdan ${ }^{3}$, Ali Abualhaija'a ${ }^{3}$, \\ Omar Alsaeidi ${ }^{3}$, Jamal Masad Melhem², Julide Sagiroglu ${ }^{3}$, Orhan Alimoglu²
}

Received: 27 Apr 2019

Published: 2 Jul 2020

\section{Abstract}

Background: Multiple risk factors contribute to the development of breast cancer, including age, positive family history, early menarche, late menopause and the strongest factor being female gender. In this study, we aimed to investigate the proportion of breast cancer patients with certain risk factors, the prevalence of each cancer type, in addition to the surgical procedures performed.

Methods: The medical records of patients diagnosed with breast cancer from January 2010 to November 2015 were evaluated retrospectively regarding demographics, breast cancer risk factors, comorbidities, diagnostic methods, tumor location, cancer type and stage, pathological findings, tumor markers, harvested lymph nodes and the types of surgical procedures. The collected data were statistically analyzed as number, mean, and frequency as percentages. Cases with deficient medical records were excluded from the analysis of certain parameters.

Results: The sample consisted of 120 patients, 118 (98.3\%) of whom were women. The mean age was $56.5 \pm 12.0$ years. The most common diagnostic method at presentation was self-exam in $93.3 \%$ of patients. Invasive ductal carcinoma was the most common type of tumor $(80.0 \%)$. The pathological stages could be determined for only 106 patients, and 26 patients $(24.5 \%)$ were at stage 1 disease, 45 patients $(42.5 \%)$ were at stage 2 whereas 34 patients (32.1\%) were at stage 3. According to the results of pathological examinations, $72.6 \%$ ( 85 patients) of the cases were estrogen receptor positive, $61.2 \%$ (71 patients) were progesterone receptor positive while $24.8 \%$ (27 patients) were HER positive. Modified radical mastectomy (MRM) was performed in $52(43.3 \%)$ patients and wide local excision (WLE) was preferred in $46(38.3 \%)$ cases.

Conclusion: Advanced age, positive family history, and prolonged estrogen exposure were remarkable in the majority of patients. Moreover, the most common type of breast cancer was invasive ductal carcinoma, and around half of the patients presented at stage 2 disease. Modified radical mastectomy and WLE were the most commonly performed surgical procedures.

Keywords: Breast Cancer, Epidemiology, Jordan

Conflicts of Interest: None declared

Funding: None

*This work has been published under CC BY-NC-SA 1.0 license.

Copyright $₫$ Iran University of Medical Sciences

Cite this article as: Halbony H, Salman Kh, Alqassieh A, Albrezat M, Hamdan A, Abualhaija'a A, Alsaeidi O, Masad Melhem J, Sagiroglu J, Alimoglu O. Breast cancer epidemiology among surgically treated patients in Jordan: A retrospective study. Med J Islam Repub Iran. 2020 (2 Jul);34:73. https://doi.org/10.47176/mjiri.34.73

\section{Introduction}

Breast cancer is the leading type of cancer in females both worldwide and in Jordan (1).

Corresponding author: Dr Hala Halbony, hala.halbouni@yahoo.com

1. University of Jordan, Faculty of Medicine, Amman, Jordan

2. University of Jordan, General Surgery Department, Amman, Jordan

3. Istanbul Medeniyet University, Goztepe Research and Training Hospital, General Surgery Department, Istanbul, Turkey
Genetic and environmental factors, including diet, behaviors such as exercise as well as radiation exposure, lactation, oral contraceptives, obesity, and parity seem to be

\section{$\uparrow$ What is "already known” in this topic:}

Most of the risk factors contributing to the development of breast cancer, in addition to the histological types are already known.

\section{$\rightarrow$ What this article adds:}

This article adds to literature the statistical evaluation of the most common presentation of breast cancer in patients, the stage at presentation, in addition to the surgical management done to these patients in a middle-eastern country. 
in close relation with the pathogenesis of breast cancer. Individual differences may be a hedge before any interventions that can potentially prevent breast cancer (2).

Early breast cancer is usually asymptomatic, and pain and discomfort are generally absent. Early diagnosis is mandatory in preventing breast cancer. Nowadays, mammography, ultrasound, and magnetic resonance imaging are the main modalities for the diagnosis. On the other hand, biomarkers provide an opportunity for diagnosis of the breast cancer (3).

Breast cancer treatment is shaped predominantly by awareness of breast cancer. The disparity in the quality of care stems mainly from different management modalities of healthcare institutions, as well as various socio-demographic factors. Younger populations and patients with higher education are more likely to insist on the latest methods of breast cancer diagnosis (4).

Following the confirmation of the pathology, management options are considered.

Surgery, along with radiation therapy, adjuvant hormone and/or chemotherapy is the primary treatment for breast cancer. Radiation therapy following surgery has been shown to be very effective in reducing recurrence. Hormone-receptor positive tumors have an excellent response to hormonal therapy, while axillary dissection provides excellent local control in patients diagnosed for early breast cancer with axillary metastasis.

In the present study, we aimed to investigate the proportion of breast cancer patients with certain risk factors, the prevalence of each cancer type, in addition to the surgical procedures performed.

\section{Methods}

After the approval of the local ethics committee, the medical records of patients diagnosed with breast cancer in Jordan University Hospital, Amman, Jordan, from January 2010 to November 2015 were evaluated retrospectively regarding demographics (age, gender), breast cancer risk factors (family history, age of menarche and menopause), comorbidities, diagnostic methods (self-examination, mammography or incidental diagnosis), tumor location, cancer type and stage, additional pathological findings (lymphovascular invasion, estrogen receptor, progesterone receptor, and HER status), tumor markers, harvested lymph nodes and the types of surgical procedures. The collected data were statistically analyzed as number, mean, frequency as percentages, and statistical analyses were conducted using SPSS Version 22. Cases with deficient medical records were excluded from the analysis of certain parameters.

\section{Results}

The study group of 120 patients consisted of 118 women (98.3\%) and 2 men (1.7\%). The mean age of 115 patients was $56.5 \pm 12.0$. Family history could be determined, in 89 patients and was positive in $21.3 \%(\mathrm{~N}=19)$ while negative in $78.7 \%(\mathrm{~N}=70)$. The mean age of menarche in our sample was 13.28 , whereas the mean age for menopause was $50 \pm 2.6$ years.

We could detect comorbidities in $67.5 \%(81 / 120)$ of our sample and they were diabetes mellitus in $29.6 \%(24 / 81)$, hypertension in $35.8 \%(29 / 81)$, ischemic heart disease in $6.2 \%(5 / 81)$ and other comorbidities in $28.4 \%(23 / 81)$ of patients.

The presentation of the first diagnostic finding was determined in 104 patients. The most common method was self-exam in $93.3 \%(\mathrm{~N}=97)$. Other methods were mammogram in $5.8 \%(\mathrm{~N}=6)$ and incidental in $0.96 \%(\mathrm{~N}=1)$.

Out of 99 patients $83(83.8 \%)$ patients underwent tru-cut biopsy and $16(16.2 \%)$ did not.

Right-sided tumors were found in $55 \%(\mathrm{~N}=66)$ of the patients, left-sided tumors in $41.7 \%(\mathrm{~N}=50)$, and bilateral tumors in $3.3 \%(\mathrm{~N}=4)$ of the patients.

The type of cancer could be determined in 115 patients. Invasive ductal carcinoma was the most common type $(80 \%, \mathrm{~N}=92)$. The other types are shown in Table 1 .

The pathological stage was determined for 106 patients (Table 2). The most common presenting stage was stage II $42.5 \%(\mathrm{~N}=45)$ followed by stage III $32.1 \%(\mathrm{~N}=34)$.

Out of 100 patients, $38 \%(\mathrm{~N}=38)$ had lymphovascular invasion, and $62 \%(\mathrm{~N}=62)$ the invasion was absent.

In addition, the presence of estrogen, progesterone, and HER receptors was determined in 117,116 and 109 patients, respectively. $72.6 \%(85 / 117)$ were estrogen receptor positive while $27.4 \%$ (32/117) were negative. $61.2 \%$ (71/116) were progesterone receptor positive while $38.8 \%$ (45/116) were negative. Furthermore, 24.8\% (27/109) were HER receptor positive and $75.2 \%(82 / 109)$ were negative.

According to the data regarding the histopathology in 106 patients, CA15-3 serum level in 90 patients and the number of lymph nodes in 113 patients were determined and collected from patients' files. The mean for histopathology was $3.29 \pm 6.28$, the mean for CA 15-3 was $21.48 \pm 17.81$ and the mean for the number of lymph nodes was $17.15 \pm 7.33$.

Data regarding the type of surgery was collected for all patients $(\mathrm{N}=120)$ (Table 3$)$. The most common type is

\begin{tabular}{lc} 
Table 1. Tumor types & \\
\hline Type of cancer & Frequency \\
\hline Invasive ductal carcinoma & $92(80 \%)$ \\
Ductal carcinoma in situ & $7(6.1 \%)$ \\
Invasive lobular carcinoma & $6(5.2 \%)$ \\
Invasive muscinous carcinoma & $7(6.1 \%)$ \\
Intracystic papillary carcinoma & $1(0.9 \%)$ \\
Invasive tubular carcinoma & $2(1.7 \%)$ \\
Total & $115(100 \%)$ \\
\hline
\end{tabular}

Table 2. The pathological stages

\begin{tabular}{lc}
\hline Pathological stage & Frequency \\
\hline I & $1(0.9 \%)$ \\
I & $26(24.5 \%)$ \\
II & $45(42.5 \%)$ \\
III & $34(32.1 \%)$ \\
Total & 106 \\
\hline & \\
Table 3. Data regarding type of surgery & Frequency \\
\hline Type of surgery & $6(5.0 \%)$ \\
Mastectomy & $52(43.3 \%)$ \\
Modified radical mastectomy & $6(5.0 \%)$ \\
Nipple Sparing Mastectomy & $10(8.3 \%)$ \\
Skin Sparing Mastectomy & $46(38.3 \%)$ \\
Wide local excision & 120 \\
Total &
\end{tabular}


MRM 43.3\% ( $\mathrm{N}=52)$ followed by WLE 38.3\% $(\mathrm{N}=46)$.

Finally, out of 113 patients, 112 (99.1\%) underwent axillary dissection and one $(0.9 \%)$ did not.

\section{Discussion}

The high mortality of breast cancer can be attributed to the delay in medical care which in some cases may be due to illiteracy, poor access to diagnostic services in rural areas, marital status, in addition to the stigma of cancer diagnosis (5). Our findings are compatible with worldwide results including a study that was conducted in Manitoba, where the mean age of diagnosis lies between the age of 50 and 69 years (6). It is suggested that early symptoms of breast cancer in older women may be attributable to aging and menopause (5).

Consistent with the findings of previous studies, the female gender is the most predominant risk factor in breast cancer. In our study, two out of 120 patients were men. Furthermore, men tended to be older than women, and this may be attributable to the fact that the etiology of male breast cancer is not completely understood. There are no routine screening guidelines for men, which would delay the diagnosis leading to a worse prognosis (7).

Age at menarche and natural menopause are complex conditions with high heritability. Usually, early menarche or late menopause is associated with an increased risk of breast cancer (8). The analysis of data in our study shows that the mean age of menarche in our sample was 13.28 , which is consistent with the results of studies which revealed that women who had their menarche between the ages of 14 and 15, are protected from breast cancer by $54 \%$ (9). The mean age for menopause was 50 years, which is also consistent with the results of other researches that categorize women with menopause at 50 or above as high-risk group (10).

In this study, only 10 patients out of 120 patients stated to have a positive family history of breast cancer, and this may be attributable to the social factors in the Arab world where some consider cancer as a stigma.

Regular screening for breast cancer, as mentioned in the scientific literature, facilitates prompt detection of breast cancer and hence early intervention which potentially leads to lower mortality rates (11). Out of the 120 studied cases, one case was incidentally detected, six cases were detected by routine mammogram screening, and 97 cases were by self-exam. Studies reveal that younger women are more symptomatic than the older generation (12).

The most common type of breast cancer is reportedly invasive ductal carcinoma (IDC) (13), and together with invasive lobular carcinoma, they comprise up to $95 \%$ of all breast cancers (14). Our study has shown similar results, where 92 out of 120 patients were diagnosed with IDC and six with ILC.

Breast-conserving and oncoplastic surgery are becoming the most popular interventions in breast cancer. Sentinel lymph node biopsy in the management of the axilla is primary, and selected patients undergoing conservative mastectomy also receive intra-operative irradiation of the nipple-areola complex (15). Our data has shown that modified radical mastectomy (MRM) is the most popular surgical procedure for breast cancer $(43.3 \%)$, as literature states that the final cosmetic results of MRM are better than those obtained with radical mastectomy. The second most commonly performed procedure is wide local excision (38.3\%); 8.3\% underwent SSBM, 5.0\% NSBM, and 5.0\% simple mastectomy. Axillary clearance was performed for 93.3\% of the patients; these results are consistent with those of previous studies, which state that the axillary dissection is standard in breast cancer management (16).

\section{Conclusion}

Our study confirms that the risk of breast cancer is increased in the presence of the known risk factors, which other studies have emphasized as well. The results strongly agree with the need to raise awareness about breast cancer through various campaigns in order to improve outcomes of management. We accept that there were several limitations in this study, and our findings cannot be generalized beyond the study sample, and the poverty of documentation in patients' files limited the predictive power of the study.

\section{Conflict of Interests}

The authors declare that they have no competing interests.

\section{References}

1. Obeidat RF, Dickerson SS, Homish GG, Alqaissi NM, Lally RM. Controlling fear: Jordanian women's perceptions of the diagnosis and surgical treatment of early-stage breast cancer. Cancer Nurs. 2013;36(6):484-92.

2. Song M, Lee KM, Kang D. Breast cancer prevention based on geneenvironment interaction. Mol Carcinog. 2011;50(4):280-90.

3. Trecate G, Sinues PM, Orlandi R. Noninvasive strategies for breast cancer early detection. Future Oncol. 2016;12(11):1395-411.

4. Obeidat R, Khrais HI. Information needs and disclosure preferences among Jordanian women diagnosed with breast cancer. J Cancer Educ. 2015;30(1):94-9.

5. Dianatinasab M, Fararouei M, Mohammadianpanah M, ZareBandamiri M. Impact of social and clinical factors on diagnostic delay of breast cancer: A Cross-sectional Study. Medicine. 2016;95(38)

6. Decker KM, Kliewer EV, Demers AA, Fradette K, Biswanger N, Musto $\mathrm{G}$, et al. Cancer incidence, mortality, and stage at diagnosis in first nations living in Manitoba. Curr Oncol. 2016;23(4):225-32.

7. Wu Q, Li J, Zhu Sh, Wu J, Li X, Liu Q, et al. Poorer breast cancer survival outcomes in males than females might be attributable to tumor subtype. Oncotarget. 2016 Sep 15.

8. Shi J, Zhang B, Choi JY, Gao YT, Li H, Lu W, et al. Age at menarche and age at natural menopause in East Asian women: a genome-wide association study. Age (Dordr). 2016

9. Laamiri FZ, Hasswane N, Kerbach A, Aguenaou H, Taboz Y, Benkirane $\mathrm{H}$, et al. Risk factors associated with a breast cancer in a population of Moroccan women whose age is less than 40 years: a case control study. Pan Afr Med J. 2016;24:19.

10. Mansha PM, Saleem M, Wasim M, Tariq M. Prevalence of Known Risk Factors in Women Diagnosed with Breast Cancer at Inmol Hospital, Lahore. Asian Pac J Cancer Prev. 2016;17(2):563-8.

11. Roder D, Farshid G, Gill G, Kollias J, Koczwara B, Karapetis C, et al. Breast cancer screeningopportunistic use of registry and linked screening data for local evaluation. J Eval Clin Pract. 2016.

12. Partridge AH, Hughes ME, Ottesen RA, Wong YN, Edge SB, Theriault RL, et al. The effect of age on delay in diagnosis and stage of breast cancer. Oncologist. 2012;17(6):775-82.

13. Jemal A, Bray F, Center MM, Ferlay J, Ward E, Forman D. Global cancer statistics. Cancer J Clin. 2011;61(2):69-90.

14. Engels CC, Fontein DBY, Kuppen PJK, Kruijf EM, Smit VTHBM, 
Breast cancer epidemiology

Nortier JWR, et al. Immunological subtypes in breast cancer are prognostic for invasive ductal but not for invasive lobular breast carcinoma Br J Cancer. 2014;111(3):532-8.

15. Benson JR, Dumitru D, Malata CM. Oncologic safety of conservative mastectomy in the therapeutic setting. Gland Surg. 2016;5(1):37-46.

16. Houvenaeghel G, Boher JM, Reyal F, Cohen M, Garbay JR, Classe $\mathrm{JM}$, et al. Impact of completion axillary lymph node dissection in patients with breast cancer and isolated tumour cells or micrometastases in sentinel nodes. Eur J Cancer. 2016;67:106-118. 